\title{
Three-dimensional cell cultures as a new tool in drug discovery
}

\author{
KARMEN BRAJŠA* \\ MARIJA TRZUN \\ IVO ZLATAR \\ DUBRAVKO JELIĆ \\ Pharmacology in vitro, Fidelta Ltd. \\ Prilaz baruna Filipovića 29, HR-10000 Zagreb \\ *Correspondence: \\ Karmen Brajša \\ e-mail:karmen.brajsa@glpg.com
}

Key words: new anticancer drugs, in vitro assays 2D and 3D cell cultures, 3D cell cultures technologies, applications in drug discovery

\section{Abbreviations \\ 2D - Two - dimensional cell cultures \\ 3D - Three - dimensional cell cultures \\ NCE - new chemical entity \\ $\mathrm{PZ}$ - proliferation zone \\ QZ - quiescent or hypoxic zone \\ $\mathrm{NZ}$ - necrotic or apoptotic cells zone}

Received January 25, 2016

Revised February 29, 2016.

Accepted March 03, 2016.

\begin{abstract}
Background and purpose: Producing of reliable information about pharmacological activity of new chemical entities is essential in early stages of drug discovery and development. There is a continuous need for improvement of existing in vitro technologies, in order to get more accurate and more predictive biological data (and for compounds selection) in pre-clinical screening methods and models.
\end{abstract}

Materials and methods: Two-dimensional (2D) cell cultures, in comparison with original tissues, does not fully reproduce in vivo cell growth and differentiation. Therefore, significant efforts have been made toward the development of more realistic three-dimensional (3D) in vitro cell culture models that would better mimic tissue physiology.

Results: Two-dimensional (2D) cell cultures, in comparison with original tissues, does not fully reproduce in vivo cell growth and differentiation. Therefore, significant efforts have been made toward the development of more realistic three-dimensional (3D) in vitro cell culture models that would better mimic tissue physiology. Basic concepts and advantages of $3 D$ cell cultures, as well as different approaches in technologies that enable the cell growth in $3 D$ will be presented here. Possible applications of $3 D$ cell culture in drug discovery will be discussed, and example of formation of spherical growth of three different human breast cancer cells (MDAMB-231, SK-BR-3 and T-47D cells) in 3D format will be shown.

Conclusions: Although biological significance of obtained data from $2 D$ and $3 D$ cell cultures is still poorly understood, discrepancy of compunds activity illustrated importance of implementation $3 D$ cell culture assays in early part of drug discovery process.

\section{INTRODUCTION}

T he costs for successfully introduction a NCE (new chemical entity) drug compound to the market is between $\$ 800$ million and $\$ 1,2$ billion, with an average duration of about $10-15$ years. Expenses are even much higher in the case of late stage failure (or failure of already marketed drug) (1). Analyses of clinical trials reveal that about $67 \%$ of drug lead molecules fail in late clinical trials stages, most of them due to poor efficacy and safety issues. This high attrition rate indicate that current pre-clinical screening and in vitro models do not adequately provide critical information required for prediction of efficacy and safety issues, which remain the main reasons for drug failure (2). The ability of an assay to produce reliable information about tested NCEs is essential in drug development. Therefore, one way to improve drug dis- 
covery and the development process could be the use of new technologies in pre-clinical screening and in vitro models, in order to ensure more accurate and more predictive data that would be the main guide through the go/no-go decision on NCE selection. Limitations of twodimensional (2D) cell cultures, related to the different cell shape, biochemical features, cellular environment and morphology of the cells (in comparison with original tissue), is that they do not fully reproduce cell growth and differentiation of conditions in vivo. Therefore, in the last decade, significant efforts have been made towards the development of more realistic in vitro cell culture models which can be "near to in vivo" conditions. Three-dimensional (3D) cell cultures are the product of such efforts due to the need to work with cellular models that better mimic tissue physiology.

\section{Basic concepts and advantages of 3D cell cultures}

The term three-dimensional (3D) cell culture is applied in relation to cell growth in a 3D environment, in some kind of matrix or on a scaffold with $3 \mathrm{D}$ structure. The term is used as opposite to the flat surface of conventional two-dimensional (2D) cell cultures (3). Cell growth in $3 \mathrm{D}$ conditions is different and in many aspects advantageous over conventional $2 \mathrm{D}$ culture conditions. In $2 \mathrm{D}$ culture conditions, cells need to adapt to a flat and rigid surface that can result in altered cell metabolism, biochemical features, cell cycle kinetics, morphology, functionality and cellular and physiological responses, in comparison to the native conditions that are present in organs (4). One of the key differences between these two growth conditions is the impact of the environment on the physical shape and geometry on the cell. It has been known that cell shape and contacts between cells influence the cytoskeleton which in turn can regulate gene and protein expression and influence on cell function. Cells grown in a $3 \mathrm{D}$ environment support their natural $3 \mathrm{D}$ physical shape, most often in a form of spheroids, which facilitates cell - cell regulatory mechanisms and signaling networks.

Spheroids are formed of cells with growth in three dimensions, which are composed of cells in various growth stages placed in different layers. Viable cells, most of them in the proliferating stage, are in the outer layer due to high exposition to the medium, where they form a proliferation zone (PZ) (5) (Figure 1). Cells in the core generate a quiescent or hypoxic zone (QZ), with necrotic or apoptotic cells in the centre (NZ). The proliferating rates and morphology of 3D spheroids is dependent on cell types and the $3 \mathrm{D}$ model conditions in which the cells are cultured.

In addition to the homogeneous $3 \mathrm{D}$ cell cultures arising from single cell line, it is possible to prepare also threedimensional co-cultures with other cells and cellular components which naturally occur in their microenviron-

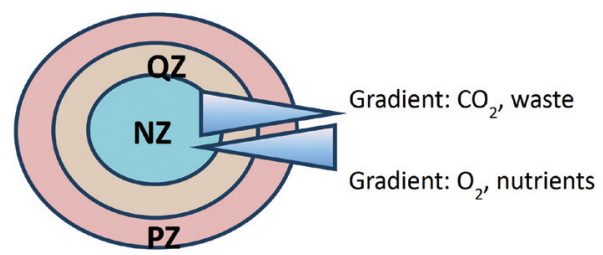

Figure 1: Spheroid cell growth composition; $P Z$ proliferation zone, $Q Z$ quiescent or hypoxic zone, $N Z$ necrotic zone.

ment, and mimic complex organ-like structures which may be more clinically relevant. Use of heterogeneous cultures with several cell types is suitable for co-cultures and cell-extracellular matrix interactions discovery. Such microenvironment supports the growth, proliferation and migration of cells through a network of signals propagated by interactions that include the extracellular matrix, other cells, growth factors, chemokines and cytokines.

Experimental tissue modeling has been very successful in mimicking structure and function of normal organs (like liver, for example), by using co-cultures of cells of different histological origin, such as hepatocytes and fibroblasts. In contrast, efforts at modeling solid tumors containing cells with various histologies and functions have remained sparse to date (5). Multicellular 3D culture systems containing more than one cell type and exhibiting formation of a complex extracellular matrix represent a more physiologically relevant environment, yet provide a challenge and unmet need for in vitro pharmacological assay methods originally designed for measuring events from monolayers of cells.

\section{D cell culture technologies}

There are many different approaches and technologies that enable the cell growth in 3D. A variety of new assay formulations, multiplexing and staining methods are designed and validated for 3D cell culture models and microtissues, which are based on measuring of ATP for cell viability, DNA staining of dead cells, caspase markers of apoptosis, glutathione as marker of oxidative stress, or luciferase reporter expression, and further efforts are aimed in validation other assays and technologies using hanging drops, hydrogels, $\mu$-fluidic assay chips and synthetic scaffolds (6).

$3 \mathrm{D}$ culture systems are classified as either scaffold-free or scaffold-based (natural or synthetic). Many companies provide scaffold-based 3D culture systems, with a huge variety of materials used in these technologies. More than 100 types of matrices and scaffolds (organic and inorganic) are currently in use. Some of these products are designed to improve the accuracy of in vitro assays in a routine and cost-effective manner and therefore have application in laboratories on daily basis. Two different techniques can be distinguished here: i) cell-seeding on an 
acellular 3D matrix and ii) dispersion of cells in a liquid hydrogel, followed by polymerization (7). One of most important characteristics of material used for $3 \mathrm{D}$ cell growth is their level of rigidity. The rigidity of matrix affect extends to cell contraction. If the matrix is rigid, it is more difficult for the cells to contract, followed by stress fiber formation in cells and different cell function. Based on their rigidity, 3D materials can be classified into three categories: hydrogels, microporous ,spongy” scaffolds and scaffold-free 3D spheroids or cellular aggregates (8).

Hydrogels possess high water content and cross-linked natural material (agarose, fibrin, collagen, laminin), which provide „soft” environments for cell growth. 3D materials based on hydrogels are used for a broad tissues types such as bone, cartilage and nervous. One of the very popular product of this group is Matrigel ${ }^{\mathrm{TM}}$ (9).

„Spongy” scaffolds materials are polystyrene, poly-LLactide Acid or Poly (lactic-co-glycolic) acid (PLGA). The major advantages of these scaffolds are defined geometry and fixed size of pores (10).

The generation of scaffold-free microtissue spheroids by gravity-enforced self-assembly in hanging drops, forcedfloating, or agitation-abased approaches has been used for decades, with particular versatility and interest in this type of the 3D culture system in the last decade (7).

Among others, scaffold-free 3D cell cultures produced with hanging drop configuration are one of the most convenient for in vitro screening usage. This method used a small aliquot of single cell suspension pipetted in the wells forming of drops and cells accumulate at the bottom of the drops, proliferate and form spheres. The hanging drop method is simple and has been reported to have a reproducibility of almost 100\% (11).

Such 3D spheroid culture techniques are today adapted and applicable for both basic research and highthroughput screening (HTS). Design of 96-well or 384well microtitre plates used for scaffold-free 3D culture assays has become increasingly relevant for the pharmaceutical industry.

\section{Applications of 3D cell culture in drug discovery}

During the 1960s and 1970s, drug discovery was based largely on screening of chemical libraries of synthetic small molecules, natural products or extracts in either animal models, or organ systems, to identify substances that had a desirable therapeutic effect. Since the early 1990s, the target-based drug discovery paradigm has become the dominant approach in the pharmaceutical industry. This process is characterized by high-throughput screening of large compound libraries against isolated biological targets which are thought to be disease-related. Due to the suboptimal success of the target-based approach, a tendency towards screening in, so-called, phe- notypic assays has reappeared in the last decade. Phenotype-based drug discovery generates molecules that produce biological effects in the biological system irrespective of their mode of action and the hit screening is performed with in vitro cell-based systems (phenotypic assays), and later with in vivo animal models that mimic specific aspects of disease (12). Today, phenotypic assays are connected with high content screening (HCS), a platform that measures multiple parameters within the cell, and relationships between the analysed parameters. Furthermore, 3D multicellular cultures have potential to improve phenotypic assays providing more relevant biological information in such a set up. Today, drug discovery screening continues to complement HTS with HCS and $3 \mathrm{D}$ cell culture technology, which will become essential for setup and development of more relevant in vitro assays (13).

Due to better correlation with in vivo conditions, 3D cultures have potential in the early phase of the drug discovery process, especially in cell-based screening. The main applications are in research of stem cells, primary cells, human cell lines, cancer cells, growth factor release, and tumor xenografts. The most successfull improved types of such assays with 3D cultures are cell viability, proliferation, cell migration and signalling (14).

It was shown that $3 \mathrm{D}$ cell cultures have different gene and protein expression in comparison with $2 \mathrm{D}$ cell cultures. Zschenker et al. compared gene expression levels in different cancer cell lines and their tissue origins and concluded that more than $30 \%$ of genes have different expression level and this is a very important issue in target-oriented screening (15). Also, proliferation rates of cells cultured in 3D conditions are lower in comparison to proliferation rates of same cells grown in 2D conditions. However, this depends on the 3D model and conditions of cell growth. Also, differences in cell viability were observed. In short term cell cultures (up to 5 days) cell viability is the same in $2 \mathrm{D}$ and in $3 \mathrm{D}$ growth conditions. In long term cell cultures cell viability in $3 \mathrm{D}$ conditions were slightly reduced probably due to the different exposure of cell area to the environment and dye uptake (16). To analyse early drug-drug interaction, human hepatocytes are the recommended in vitro assay system. The problem with such an assay is time limited Cytochrome P450 (CYP) activity and stability. It was shown that $3 \mathrm{D}$ human hepatocyte cell cultures have advantage over 2D cell cultures because viability of hepatocytes together with CYP activity is longer and more stable (17). The largest number of drug lead molecule failures is in the oncology field. One of the main reasons of high drug attrition rate in oncology is that standard tools that cancer biologists use as 2D cell cultures in vitro have poor clinical predictive power (18). Conventional two-dimensional (2D) cell cultures are not able to completely describe malignant phenotypes that are related to tumorogenicity in vivo. In $2 \mathrm{D}$ culture conditions cells need to adapt to a flat and rigid surface 


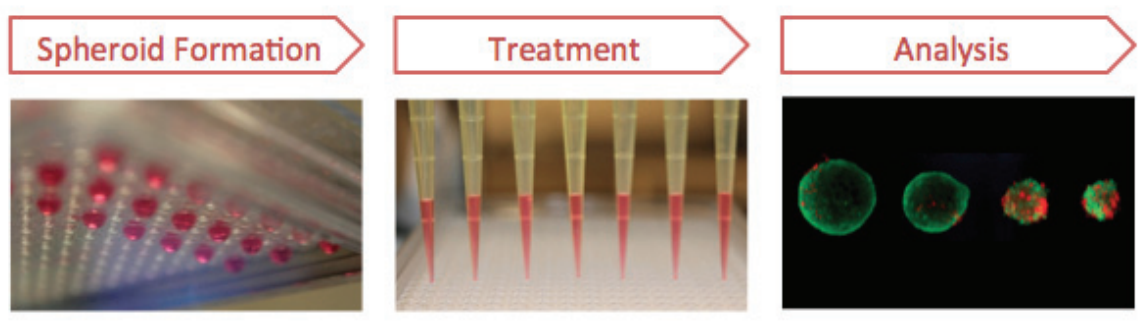

Figure 2: 3D Biomatrix hanging drop plates used for $3 D$ spheroid formation, treatment and analysis. Pictures taken from Perfecta $3 D^{\oplus} H a n g i n g$ Drop Plates Protocols (https://3dbiomatrix.com/).

that can result in altered cell metabolism, functionality and pharmacological respond (4). Therefore, three-dimensional $(3 \mathrm{D})$ cell culture techniques have a valuable impact in the oncology field. Tumor spheroids are well established in basic cancer research and experimental therapeutics. Using these 3D cultures, significant progress has been made in understanding the regulation of invasion and metastasis, of angiogenesis, and of cell cycle (5). Larger spheroids contain, along with other layers, areas of necrotic cells at the centre and are useful for influence of hypoxia in cancer research (Figure 1) (19). Evaluation of drug resistance and sensitivity generally showed that tested drugs showed different IC50 values in 3D cell culture assay in comparison with IC50 values obtained in 2D assay (20).

Among other reasons, differences of $2 \mathrm{D}$ and $3 \mathrm{D}$ cell response can be also due to spatial organisation of surface receptors, cancer gene expression, and difference in cell stages, drug acceptability and $\mathrm{pH}$ gradient in spheroids. Nevertheless, results obtained in 3D cancer cells are more in line with in vivo models than results obtained in 2D, especially if patient-derived primary tumour cells are used rather that commercial cell lines (21).

Among various 3D methods, the hanging drop method is one of the best characterised models for $3 \mathrm{D}$ culture, with all its advantages and disadvantages (11). In our laboratory, we have excellent experience with 3D Biomatrix hanging drop plates (http://3dbiomatrix.com/, Figure 2). They support production of $3 \mathrm{D}$ cell cultures of broad range of different cell lines, and enable produced 3D cultures to be tested in a high-throughput manner (22). Also, because it is a scaffold free system, they eliminate the possibility of interaction of the NCE with scaffold material.

Among others, in such systems we tested amidino substituted benzimidazole and benzimidazo $[1,2-a]$ quinoline derivatives (22 compounds) designed as a compounds with antitumor activity as a small platform for comparison of antitumor activity in 2D and 3D cell culture systems and for correlation with structure-activity relation-

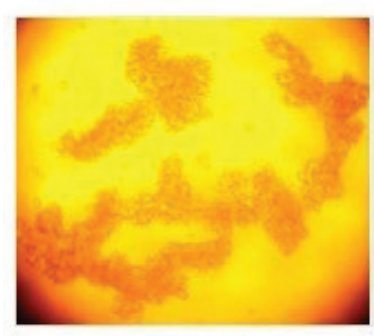

*MDA-MB-231_Day 1_5x

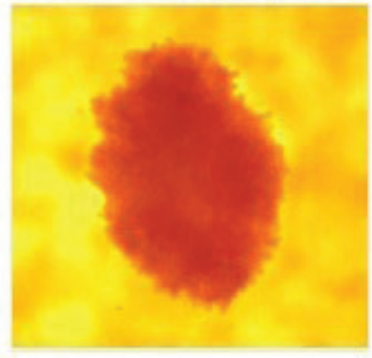

* MDA-MB-231_Day 4_5x

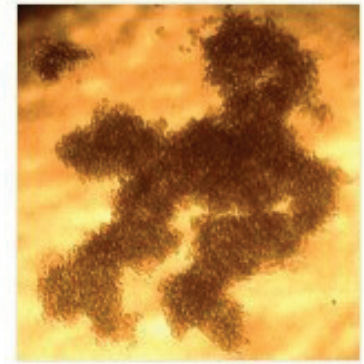

*MDA-MB-231_Day 2_4x

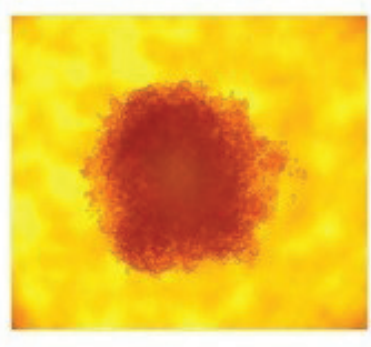

* MDA-MB-231_Day 5_5x

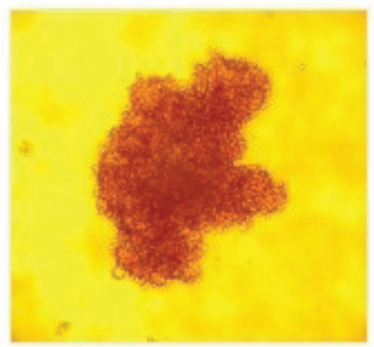

*MDA-MB-231_Day 3_5x

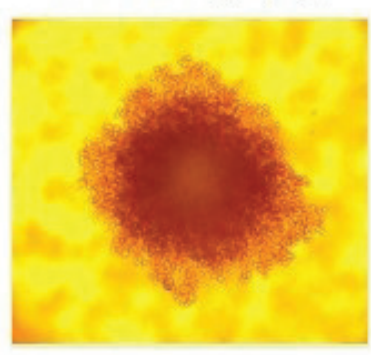

*MDA-MB-231_Day 6_5x

Figure 3: Formation of spherical growth of $M D A-M B-231$ cells in 3D format during period of 6 days (4-5x magnification, seeding density 5000 cells/well). Pictures are taken in Fidelta Ltd., by using $3 D$ Biomatrix hanging drop plates. 


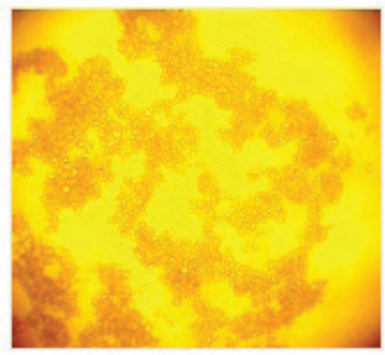

*SK-BR-3_Day 1_5x

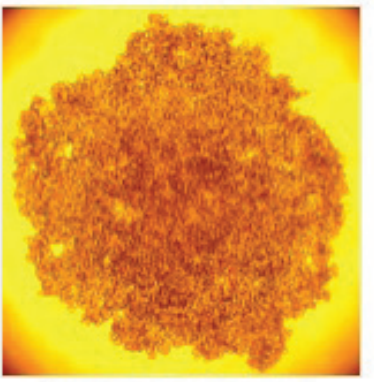

* SK-BR-3_Day 4_5x

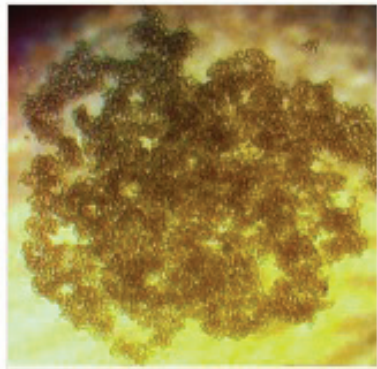

* SK-BR-3_Day 2_4x

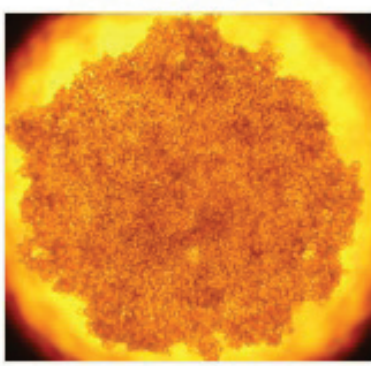

* SK-BR-3_Day 5_5x

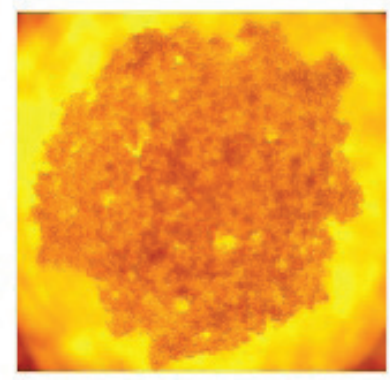

* SK-BR-3_Day 3_5x

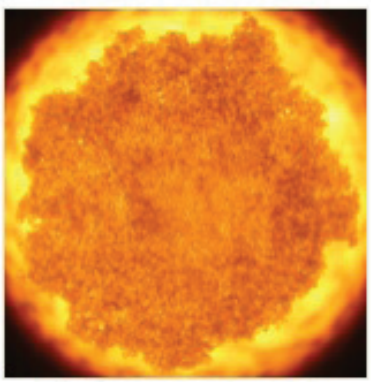

* SK-BR-3_Day 6_5x

Figure 4: Formation of spherical growth of SK-BR-3 cells in 3D format during period of 6 days (4-5x magnification, seeding density 5000 cells) well). Pictures are taken in Fidelta Ltd., by using $3 D$ Biomatrix hanging drop plates.

ship (SAR). 3D hanging-drop cell culture method was applied on three human cancer breast cell lines (SK-BR-3, MDA-MB-231 and T-47D) and two pancreatic cancer cell lines (MIA PaCa-2 and PANC-1). As standard drugs we tested doxorubicin, docetaxel, vinorelbine and gemcitabine. All cell lines showed increased chemoresistance in 3D cell growth to standard drugs and for some com- pounds from our platform we have observed significant disagreement indicating that some prominent compounds can be discarded in the early phase of research, while a chance was given to compounds with false positive result (23). We concluded that, as well as in $2 \mathrm{D}$ assay, the shift in $\mathrm{IC}_{50}$ in a $3 \mathrm{D}$ cell culture models is not dependent only on the mode of action of the tested compounds, but it is

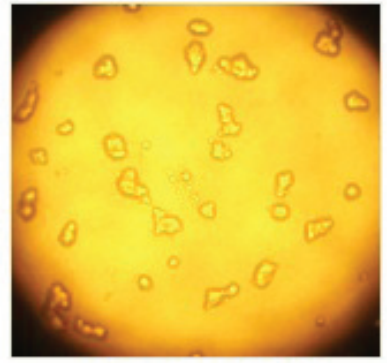

* T-47D_Day 1_5x

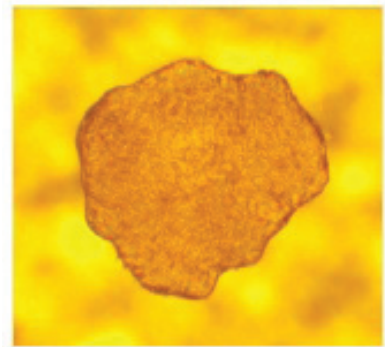

* T-47D_Day 4_5x

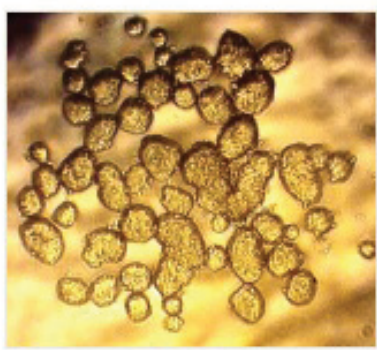

*T-47D_Day 2_4x

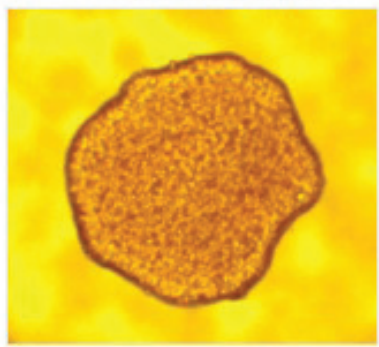

* T-47D_Day 5_5x

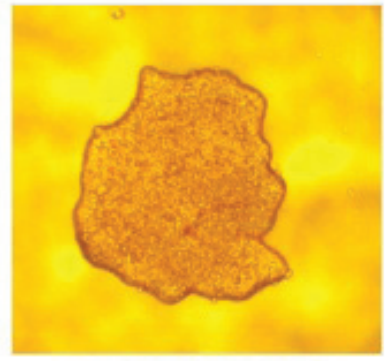

*T-47D_Day 3_5x

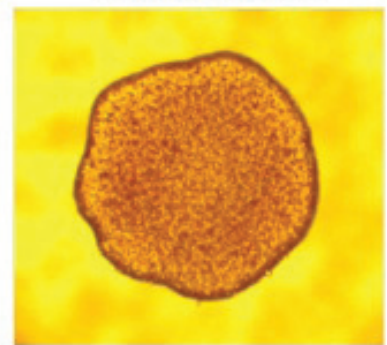

*T-47D_Day 6_5x

Figure 5: Formation of spherical growth; T-47D in 3D format during period of 6 days (4-5x magnification, seeding density 5000 cells/well). Pictures are taken in Fidelta Ltd., by using $3 D$ Biomatrix hanging drop plates. 
also affected by the cell line sensitivity and (in case of 3D cultures) spheroid stability, and cell line growth have an impact on the cell line sensitivity. Examples of day-to-day formation of $3 \mathrm{D}$ spherical growth for different tumor cell lines, by using Perfecta3D hanging drop plates which are easy-to-use multi-well plates that facilitate uniform spheroid formation, are presented on Figures 3, 4, and 5, where optimal timing needed for ideal spheroid formation could be very nicely recognized for every particular cell line.

\section{Limitations of 3D cell cultures}

Despite the above mentioned advantages and tremendous progress made to adjust 3D culture technologies for pharmaceutical applications, there are still some limitations in 3D cell cultures methods, which still fail to provide relevant, standardized and validated $3 \mathrm{D}$ cell/tissue models. The ideal method for 3D cell culture is not yet optimised due to lack of a quantifiable entity of biomarkers of three dimensionality. That means that $3 \mathrm{D}$ cell cultures should have description of at least of two factors: spatial that describes dimensions, structure and spheres compactibility; and some biochemical biomarkers which would suggest composition of the microenviroment that ensure physiologically relevant cell response. There are also limitations that are specific for method type as variability of cell size, viability and number of cells per well, nonequal exposure of cells in well to drug activity, time consuming plate preparation, lack of specific equipment, etc. For scaffold-based cell culture systems, reproducibility between different batches is still unsatisfactory, and potential interactions of screening compounds with the scaffold, which is influenced also with the compound properties (hydrophilic or hydrophobic). In summary, the benefits of $3 \mathrm{D}$ cell culture system in terms of biological relevance (especially for pharmaceutical applications) is obvious, but there are still many hurdles and unmet needs, which prevent universal standardization of $3 \mathrm{D}$ culture systems for drug development (7)

\section{CONCLUSION}

The on-going trend is moving toward creating new therapies which should be more effective, reliable and safe, but on the other hand they should be less costly. However, credibility of the pharmaceutical industry and academic researchers continue to erode in advocating their traditional pipeline development strategies yielding only modest drug innovation successes. New trends in industrial drug development should use innovative and more improved new technologies in the drug development process. Those novel trends in experimentation with cells include attempts to obtain and maintain functional differentiation of human cells similar to those in vivo. Primary tissues (healthy or diseased) or isolated early passage primary cells are cultured in so-called 'ex vivo' cultures. Significant efforts are made to co-culture primary cells with the hope of at least partially simulating the in vivo environment. On the other hand, established cell lines can be cultured on specially manufactured growth surfaces (hollow fibre bioreactor, cellular multilayers on porous membranes), matrices (matrix embedded culture) and in controlled heterologous monolayer systems, as well as $3 \mathrm{D}$ cultures (multicellular spheroids), moving away from „flat biology” (24). The data obtained are integrated and analysed using systems biology and biomapping approaches. All in all, the pendulum is moving from historically highly reductionist approaches in in vitro biology towards mimicry of the in vitro and in vivo environment. Stem cells and associated in vitro organ formation, dedicated to in vitro compound testing, as well as research on their potential for therapeutic application, are creating a seed for the fast growth of regenerative medicine.

All these novel approaches will definitely aid the better understanding of multi-cellular in vivo biology and will contribute significantly towards improvement of the inherently high attrition rate of drug discovery $(25,26)$. Therefore, use of traditional 2D cell cultures and persistence of them as cell screening for NCE toxicity and efficacy is questionable. Cells grown in a $3 \mathrm{D}$ model have proven to be more physiologically relevant: they have a longer lifespan, their growth is undisturbed (without regular trypsinisations), and expressed genotype as well as phenotype are more relevant to in vivo conditions. Based on all the above mentioned differences between traditional 2D cell cultures and 3D spheroid cultures, three-dimensional cell cultures in a form of multicellular spheroids should become mandatory test systems in therapeutic screening programs.

\section{LITERATURE:}

1. Kola I, Landis J 2004 Can the pharmaceutical industry reduce attrition rates? Nature Rev Drug Dis 3:711-715 http://dx.doi.org/10.1038/nrd1470

2. Kim JB 2005 Three-dimensional tissue culture models in cancer biology Seminars in cancer biology 15:365-377 http://dx.doi.org/10.1016/j.semcancer.2005.05.002

3. Comley J 2013/14 Progress made in applying 3D cell culture technologies Drug Discov World Winter 41-58

4. Abbott A 2003 Cell culture: biology's new dimension Nature 424: 870 http://dx.doi.org/10.1038/424870a

5. Mueller-Klieser W 2000 Tumor biology and experimental therapeutics Critical Reviews in Oncology/Hematology 36:123-139 http://dx.doi.org/10.1016/S1040-8428(00)00082-2

6. Riss TL, Valley MP, Zimprich CA, Niles AL, Kupcho KR, Lazar DF 2014 Design and Validation of Bioluminescent Assays for 3D Cell Culture Models, Promega Corporation poster, Abstract\#226

7. Rimann M, Graf-Hausner U 2012 Synthetic 3D multicellular systems for drug development Current Opinion in Biotechnology 23:803-809 http://dx.doi.org/10.1016/j.copbio.2012.01.011

8. Asthana A, Kisaalita S 2013 Biophysical microenvironment and $3 \mathrm{D}$ culture physiological relevance Drug discovery today 11-12: 533-540 http://dx.doi.org/10.1016/j.drudis.2012.12.005

9. Przyborski S 2011 3D cell culture developments in technology to improve in vitro analyses Drug Discov World Spring 67-72 
10. Gentile P, Chiono V, Carmagnola I, Hatton PV 2014 An Overview of Poly (lactic-co-glycolic) Acid (PLGA)-Based Biomaterials for Bone Tissue Engineering, Int J Mol Sci 15:3640-3659 http://dx.doi.org/10.3390/ijms15033640

11. Breslin S, O'Driscol L 2013 Three-dimensional cell culture: the missing link in drug discovery Drug Discovery Today 18:240-249 http://dx.doi.org/10.1016/j.drudis.2012.10.003

12. Jelić D, Brajša K, Banjanac M, Spaventi R, Eraković Haber V 2013 In vitro biological screening in discovery of hits and leads, Review Book, Chief Editor: Dubravko Jelić Editorial Consultant: Michael J. Parnham Publisher: Research Signpost - Transworld Research Network, Managing Editor - S.G. Pandalai 262-300

13. Justice BA, Bard NA, Feider RA 2009 3D cell culture opens new dimensions in cell-based assay Drug DiscovToday 14:102-7 http://dx.doi.org/10.1016/j.drudis.2008.11.006

14. Comley J 2010 3D cell culture: easier said than done! Drug Discovery World, Summer 11:25-41

15. Zschenker O, Streichert T, Hehlgans S, Cordes N 2012 Genomewide gene expression analysis in cancer cells reveals $3 \mathrm{D}$ growth to affect ECM and processes associated with cell adhesion but not DNA repair PLos ONE 7: e34279

http://dx.doi.org/10.1371/journal.pone.0034279

16. Bonnier F, Keating ME, Wrobel TP, Majzner K, Baranska M, Garcia-Munoz A, Blanco A, and Byrne HJ 2015 Cell viability assessment using the Alamar blue assay: A comparison of $2 \mathrm{D}$ and $3 \mathrm{D}$ cell culture models Toxicology in vitro 29:124-131 http://dx.doi.org/10.1016/j.tiv.2014.09.014

17. Larson B, Cameron G, Long D, Moeller T 2014 The effect of cell culture method on long-term primary hepatocyte cell health BioTeck Application Note 1:1-4

18. Siolas D, Hannon GJ 2013 Patient-derived tumor xenografts: transforming clinical samples into mouse models Cancer Res 73: 5315-531 httpp://dx.doi.org/10.1158/0008-5472.CAN-13-1069
19. Hirschhaeuser F, Menne H, Dittfeld C, West J, Mueller-Klieser W, Kunz-Schughart LA 2010 Multicellular tumour spheroids: A underestimated tool is catching up again, J Biotechnology 148: 3-15 http://dx.doi.org/10.1016/j.jbiotec.2010.01.012

20. Lama R, Zhang L, Naim JM, Williams J, Zhou A, Su B 2013 Development, validation and pilot screening of an in vitro multicellular three-dimensional cancer spheroid assay for anti-cancer drug testing Bioorg Med Chem 21: 922 - 931 http://dx.doi.org/10.1016/j.bmc.2012.12.007

21. Choi SY, Lin D, Gout PW, Collins CC, Xu Y, and Wang Y 2014 Lessons from patient-derived xenografts for better in vitro modeling of human cancer Adv Drug Delivery Rev 79-80: 222-237 http://dx.doi.org/10.1016/j.addr.2014.09.009

22. Tung YC, Hsiao AY, Allen SG, Torisawa YS, Ho M, Takayama S 2011 High-throughput 3D spheroid culture and drug testing using a 384 hanging drop array Analyst 136: 473-8 http://dx.doi.org/10.1039/C0AN00609B

23. Brajša K, Vujasinović I, Jelić D, Trzun M, Zlatar I, KarminskiZamola G, Hranjec M 2015 2D and 3D in vitro antitumor activity of amidino substituted benzimidazole and benzimidazo[1,2-a] quinoline derivatives, J Enzyme Inhib Med Chem 27:1-7 http://dx.doi.org/10.3109/14756366.2015.1101093

24. Bhadriraju K, Chen CS 2002 Engineering cellular microenvironments to improve cell-based drug testing Drug Discov Today 7:612-620 http://dx.doi.org/10.1016/S1359-6446(02)02273-0

25. Goodsaid FM Mendrick DL 2010 Translational medicine and the value of biomarker qualification Sci Transl Med 2:47 http://dx.doi.org/10.1126/scitranslmed.3001040

26. Wehling M (2011) Drug development in the light of translational science: shine or shade?Drug Discov Today 16:1076-1083 http://dx.doi.org/10.1016/j.drudis.2011.07.008 